HD-THEP 95-14

\title{
A NOVEL LOOK AT THE MICHAEL LATTICE SUM RULES
}

\author{
Heinz J. Rothe \\ Institut für Theoretische Physik \\ Universität Heidelberg \\ Philosophenweg 16, D-69120 Heidelberg
}

\begin{abstract}
We reconsider the derivation of the Michael lattice sum rules, which relate the energy and action stored in a flux tube of a quark-antiquark pair to the static interquark potential, and show that they require essential corrections. We then find, using the coupling constant sum rule of Karsch, that the total Minkowski field energy does not match the interquark potential, if one follows conventional notions. The implications of this result are discussed.
\end{abstract}


Monte Carlo simulations of non-abelian gauge theories are usually carried out on a lattice with equal lattice spacings in the spatial and euclidean time directions. A lattice regularization using different cutoffs for the space and time directions must of course yield the same results for physical observables in the continuum limit. A knowledge of the corresponding regularized action is required to relate thermodynamical observables to expectation values of lattice operators, since such a regularization allows one to vary independently the temperature and volume of the system [1-3]. The action then depends on two coupling constants associated with the temporal and space-like plaquettes. These couplings are functions of the spatial lattice cutoff and the anisotropy parameter $\xi$, defined as the ratio of the spatial to temporal lattice spacing $[2,3]$. When taking the continuum limit with the anisotropy parameter held fixed, physical observables, such as the interquark potential and particle masses, should not depend on $\xi$. By requiring that the string tension obtained either from the expectation value of a space-time or a space-like Wilson loop be invariant under changes in the anisotropy parameter, Karsch [3] was able to show that the sum of the derivatives of the inverse coupling constants squared with respect to the anisotropy parameter, evaluated on an isotropic lattice, is given in the continuum limit by the first coefficient in the perturbative expansion of the $\beta$-function. Furthermore, by requiring that in the continuum limit the effective action be independent of the lattice regularization chosen, he was able to determine the dependence of the couplings on the anisotropy parameter.

The formulation of $S U(N)$ gauge theories on an anisotropic lattice has been used in [4] to derive sum rules relating the potential of a quark-antiquark pair to correlators of the action, or of the chromoelectric and chromomagnetic field energy, with the Wilson loop. These lattice sum rules are known as Michael's sum rules. Comparing the action sum rule given in [4] with the corresponding sum rule in ref. [3], one finds that they disagree by a factor of 2. That the action sum rule in [4] is actually incorrect has been noted recently in [5], where the authors derive this sum rule within the framework of the continuum 
formulation. Motivated by these observations, we have reexamined the derivation of the Michael sum rules, and find that there are important corrections to both the action and the energy sum rule of ref. [4]. Using the coupling constant sum rule of Karsch [3], we then find, following conventional lore, that the sum rule relating the interquark potential to the field energy in the continuum formulation would be violated in the lattice regularized theory. The implications of this result are discussed.

Consider the ground state energy $\hat{E}_{0}$ of a quark-antiquark pair separated by a distance $\hat{R}$. Quantities denoted with a "hat" will always be understood to be measured in units of the lattice spacing. The energy $\hat{E}_{0}$ can be calculated from the expectation value of the Wilson loop with spatial and temporal extension $\hat{R}$ and $\hat{T}$, respectively:

$$
\hat{E}_{0}=-\lim _{\hat{T} \rightarrow \infty} \frac{1}{\hat{T}} \ln <W(\hat{R}, \hat{T})>.
$$

On an isotropic lattice $<W(\hat{R}, \hat{T})>$ is calculated with the action

$$
S=\hat{\beta}\left(\mathcal{P}_{\tau}+\mathcal{P}_{s}\right)
$$

where, for $S U(N), \hat{\beta}$ is defined in terms of the bare coupling constant by $\hat{\beta}=\frac{2 N}{g_{0}^{2}}$, and $\mathcal{P}_{\tau}, \mathcal{P}_{s}$ denote the contributions of the time-like and space-like plaquette variables:

$$
\begin{aligned}
& \mathcal{P}_{\tau}=\sum_{n} \sum_{\mu \neq 4}\left[1-\frac{1}{2 N} \operatorname{Tr}\left(U_{\mu 4}(n)+U_{\mu 4}^{\dagger}(n)\right)\right], \\
& \mathcal{P}_{s}=\sum_{n} \sum_{i>j}\left[1-\frac{1}{2 N} \operatorname{Tr}\left(U_{i j}(n)+U_{i j}^{\dagger}(n)\right)\right] .
\end{aligned}
$$

Here $i, j$ label the spatial directions, and $U_{\mu \nu}(n)$ is the lattice variable associated with a plaquette located in the $\mu \nu$-plane at the lattice site $n$.

The lattice energy $\hat{E}_{0}$, defined by (1), is a function of $\hat{R}$ and $\hat{\beta}$. Since the self-energy contributions to $\hat{E}_{0}$, associated with the quark and antiquark, do not depend on $\hat{R}$, they can be eliminated by considering the difference $\hat{E}_{0}(\hat{R}, \hat{\beta})-\hat{E}_{0}\left(\hat{R}_{0}, \hat{\beta}\right)$, where $\hat{R}_{0}$, is some reference $q \bar{q}$-separation. Then the subtracted $q \bar{q}$ potential is given by

$$
[V(\hat{R}, \hat{\beta})]_{\text {subtr }}=-\lim _{\hat{T} \rightarrow \infty} \frac{1}{\hat{T}}\left[\ln <W(\hat{R}, \hat{T}>]_{\text {subtr }},\right.
$$


where

$$
<W(\hat{R}, \hat{T})>=\frac{\int D U W(\hat{R}, \hat{T}) e^{-\hat{\beta}\left(\mathcal{P}_{\tau}+\mathcal{P}_{s}\right)}}{\int D U e^{-\hat{\beta}\left(\mathcal{P}_{\tau}+\mathcal{P}_{s}\right)}} .
$$

From here on we will always assume that such a subtraction has been carried out, and shall drop the subscript "subtr" for simplicity. Following [4] we now take the derivative of (4a) with respect to $\hat{\beta}$. One then obtains

$$
\frac{\partial \hat{V}(\hat{R}, \hat{\beta})}{\partial \hat{\beta}}=\lim _{\hat{T} \rightarrow \infty} \frac{1}{\hat{T}}<\mathcal{P}_{\tau}+\mathcal{P}_{s}>_{q \bar{q}-0}
$$

where the expectation value $<O>_{q \bar{q}-0}$ is defined generically by

$$
<O>_{q \bar{q}-0}=\frac{<W(\hat{R}, \hat{T}) O>}{<W(\hat{R}, \hat{T})>}-<O>
$$

Hence the $\hat{\beta}$-derivative of the potential is expressed in terms of correlators of plaquette variables with the Wilson loop. These are the correlators which have been measured in Monte Carlo simulations to determine the spatial distribution of the energy density in a flux tube connecting a quark and antiquark [6].

In the limit $\hat{T} \rightarrow \infty$, the rhs of (5a) can be further simplified. Since for $\hat{T} \rightarrow \infty$ the expectation value of a plaquette variable is invariant under a "time" translation, we have that

$$
<\mathcal{P}_{\sigma}>_{q \bar{q}-0} \underset{\hat{T} \rightarrow \infty}{\approx} \hat{T}<\mathcal{P}_{\sigma}^{\prime}>_{q \bar{q}-0}
$$

where $\mathcal{P}_{\sigma}^{\prime}$ is given by an expression of the form (3), with $n$ running over the lattice sites on the fixed time slice. With a Wilson loop extending from $n_{4}=-\frac{\hat{T}}{2}$ to $n_{4}=\frac{\hat{T}}{2}$, this time slice is conveniently chosen to be the $n_{4}=0$ plane. Then

$$
\hat{\beta} \frac{\partial \hat{V}(\hat{R}, \hat{\beta})}{\partial \hat{\beta}} \approx \hat{\beta}<\mathcal{P}_{\tau}^{\prime}+\mathcal{P}_{s}^{\prime}>_{q \bar{q}-0} \rightarrow a \sum_{\vec{x}} a^{3} \frac{1}{2}<\vec{E}^{2}(\vec{x})+\vec{B}^{2}(\vec{x})>_{q \bar{q}-0}
$$

where in the last step we have taken the naive continuum limit. Here $-\frac{1}{2} \vec{E}^{2}$ and $\frac{1}{2} \vec{B}^{2}$ are the Minkowski energy densities of the chromoelectric and chromomagnetic fields expressed in terms of the euclidean fields. 
We next use the renormalization group to cast the lhs of (7) in a form involving the potential and its derivative with respect to $\hat{R}$. In the limit of vanishing lattice spacing " $a$ " we have that

$$
\frac{1}{a} \hat{V}\left(\frac{R}{a}, \hat{\beta}(a)\right) \underset{a \rightarrow 0}{\longrightarrow} V(R)
$$

where $V(R)$ is the physical interquark potential, and the behaviour of $\hat{\beta}(a)$ as a function of the lattice spacing is given, close to the continuum limit, through the renormalization group relation

$$
a=\frac{1}{\Lambda_{L}}\left(\frac{2 N b_{0}}{\hat{\beta}}\right)^{-b_{1} /\left(2 b_{0}^{2}\right)} e^{-\frac{\hat{\beta}}{4 N b_{0}}} .
$$

Here $b_{0}$ and $b_{1}$ are given by

$$
b_{0}=\frac{11 N}{48 \pi^{2}} ; \quad b_{1}=\frac{34}{3}\left(\frac{N}{16 \pi^{2}}\right)^{2} .
$$

The invariance of the lhs of (8) with regard to changes in the lattice spacing leads to

$$
\frac{\partial \hat{\beta}}{\partial \ln a} \frac{\partial \hat{V}(\hat{R}, \hat{\beta})}{\partial \hat{\beta}}=\hat{V}(\hat{R}, \hat{\beta})+\hat{R} \frac{\partial \hat{V}(\hat{R}, \hat{\beta})}{\partial \hat{R}}
$$

where it is understood that this expression is to be evaluated for $\hat{R}=\frac{R}{a}$, and with $\hat{\beta}(a)$ determined by (9). Making use of the relation (11), equation (7) takes the following form close to the continuum limit

$$
\hat{V}(\hat{R}, \hat{\beta})+\hat{R} \frac{\partial \hat{V}(\hat{R}, \hat{\beta})}{\partial \hat{R}}=\frac{\partial \hat{\beta}}{\partial \ln a}<\mathcal{P}_{\tau}^{\prime}+\mathcal{P}_{s}^{\prime}>_{q \bar{q}-0}
$$

In the case of a confining potential, $\hat{V}(\hat{R}, \hat{\beta})=\hat{\sigma}(\hat{\beta}) \hat{R}$, this equation reduces to

$$
2 \hat{\sigma}(\hat{\beta}) \hat{R}=\frac{\partial \hat{\beta}}{\partial \ln a}<\mathcal{P}_{\tau}^{\prime}+\mathcal{P}_{s}^{\prime}>_{q \bar{q}-0}
$$

which coincides with that obtained in [3] by making use of the relations (3.7), and the equation following it in that reference. The second term appearing on the lhs of (12), which gives rise to the factor of two in (13), has been missed in [4]. Hence the Michael action sum rule is incorrect, as was also recently observed in [5], where the authors derive this sum rule within the continuum formulation of QCD. 
A second sum rule, relating the interquark potential to the Minkowski field energy, can be obtained by requiring that a lattice regularization involving different lattice spacings in the temporal and spatial directions should lead to the same physical potential as that computed from an isotropic lattice. A similar argument has been used in [3] for the string tension computed either from a time-like or a space-like Wilson loop, leading to a coupling constant sum rule which will play a key role in our discussion. On an anisotropic lattice the action involves two couplings, $\hat{\beta}_{\tau}$ and $\hat{\beta}_{s}[1-3]$, associated with the time-like and space-like plaquette contributions:

$$
S=\hat{\beta}_{\tau} \mathcal{P}_{\tau}+\hat{\beta}_{s} \mathcal{P}_{s}
$$

These couplings are conventionally considered to be functions of the spatial lattice spacing $a$ and the anisotropy parameter $\xi=a / a_{\tau}$, where $a_{\tau}$ is the lattice spacing in the temporal direction. They are usually parametrized as follows $[2,3]$

$$
\hat{\beta}_{s}=\frac{2 N}{g_{s}^{2}(a, \xi)} \xi^{-1}, \quad \hat{\beta}_{\tau}=\frac{2 N}{g_{\tau}^{2}(a, \xi)} \xi,
$$

where $g_{s}^{2}(a, 1)=g_{\tau}^{2}(a, 1)=g_{0}^{2}(a)$, and where the explicit dependence on $\xi$ is chosen in such a way that the $\xi$ dependence of $g_{\sigma}(a, \xi), \sigma=s, \tau$, arises only from quantum corrections. In the weak coupling limit $\hat{\beta}_{\tau}(a, \xi)$ and $\hat{\beta}_{s}(a, \xi)$ can be related to the bare coupling $\hat{\beta}(a)$ on an isotropic lattice $[2,3]$ by

$$
\begin{gathered}
\frac{1}{\xi} \hat{\beta}_{\tau}(a, \xi)=\hat{\beta}(a)+2 N c_{\tau}(\xi)+\mathcal{O}\left(\hat{\beta}^{-1}\right), \\
\xi \hat{\beta}_{s}(a, \xi)=\hat{\beta}(a)+2 N c_{s}(\xi)+\mathcal{O}\left(\hat{\beta}^{-1}\right),
\end{gathered}
$$

where the $\xi$-dependence of the functions $c_{\sigma}(\xi), \sigma=\tau, s$, have been studied in detail in [3].

With the action (14), the lattice potential computed from the expectation value of the Wilson loop becomes a function of $\hat{R}, \hat{\beta}_{s}$, and $\hat{\beta}_{\tau}$. We now require that in the continuum limit $a \rightarrow 0, a_{\tau} \rightarrow 0, \xi=a / a_{\tau}$ fixed, the physical potential, $V \sim \frac{1}{a_{\tau}} \hat{V}$, should not depend on the choice of $\xi$, if the couplings $\hat{\beta}_{s}$ and $\hat{\beta}_{\tau}$ are tuned with $a$ appropriately. Hence for $a \rightarrow 0$ we have that

$$
\frac{d}{d \xi}\left[\frac{\xi}{a} \hat{V}\left(\frac{R}{a}, \hat{\beta}_{\tau}(a, \xi), \hat{\beta}_{s}(a, \xi)\right)\right]=0
$$


Noting that

$$
\frac{\partial \hat{V}}{\partial \beta_{\sigma}}=<\mathcal{P}_{\sigma}^{\prime}>_{q \bar{q}-0} ; \quad \sigma=\tau, s,
$$

one finds, upon carrying out the differentiation (17), and then returning to the isotropic lattice $\xi=1$, that

$$
\hat{V}(\hat{R}, \hat{\beta})=-\left[\left(\frac{\partial \hat{\beta}_{\tau}}{\partial \xi}\right)<\mathcal{P}_{\tau}^{\prime}>_{q \bar{q}-0}+\left(\frac{\partial \hat{\beta}_{s}}{\partial \xi}\right)<\mathcal{P}_{s}^{\prime}>_{q \bar{q}-0}\right]_{\xi=1}
$$

Here $\hat{V}(\hat{R}, \hat{\beta})$ is the potential in lattice units computed on an isotropic lattice. Again it is understood that this relation only holds for $\hat{R}=R / a, \beta_{\sigma}=\beta_{\sigma}(a, \xi)$, in the continuum limit. Defining

$$
\eta_{ \pm}=\frac{1}{2}\left[\left(\frac{\partial \hat{\beta}_{\tau}}{\partial \xi}\right)_{\xi=1} \pm\left(\frac{\partial \hat{\beta}_{s}}{\partial \xi}\right)_{\xi=1}\right]
$$

expression (19) can be written in the form

$$
\hat{V}(\hat{R}, \hat{\beta})=\eta_{-}<-\mathcal{P}_{\tau}^{\prime}+\mathcal{P}_{s}^{\prime}>_{q \bar{q}-0}-\eta_{+}<\mathcal{P}_{\tau}^{\prime}+\mathcal{P}_{s}^{\prime}>_{q \bar{q}-0}
$$

where the expectation values are computed on an isotropic lattice. Making use of the action sum rule (12) one obtains

$$
\hat{V}(\hat{R}, \hat{\beta})+\eta_{+} \frac{\partial \ln a}{\partial \hat{\beta}}\left[\hat{V}(\hat{R}, \hat{\beta})+\hat{R} \frac{\partial \hat{V}(\hat{R}, \hat{\beta})}{\partial \hat{R}}\right]=\eta_{-}<-\mathcal{P}_{\tau}^{\prime}+\mathcal{P}_{s}^{\prime}>_{q \bar{q}-0}
$$

We emphasize that so far our discussion has not involved any perturbative arguments. We now want to interprete the rhs of $(22)$ in terms of a continuum physical observable. It is at this (and only this) stage where, following standard lore, we make use of the weak coupling approximations (16). From the definition (20) and the relations (16) one finds that for $a \rightarrow 0$

$$
\eta_{-} \approx \hat{\beta}(a)+N\left[c_{\tau}^{\prime}(1)-c_{s}^{\prime}(1)\right] \underset{\hat{\beta} \rightarrow \infty}{\longrightarrow} \hat{\beta}(a)
$$

where $c_{\sigma}^{\prime}$ is the derivative of $c_{\sigma}$. Hence close to the continuum limit

$$
\hat{V}(\hat{R}, \hat{\beta})+\eta_{+} \frac{\partial \ln a}{\partial \hat{\beta}}\left[\hat{V}(\hat{R}, \hat{\beta})+\hat{R} \frac{\partial \hat{V}}{\partial \hat{R}}(\hat{R}, \hat{\beta})\right] \approx \hat{\beta}<-\mathcal{P}_{\tau}^{\prime}+\mathcal{P}_{s}^{\prime}>_{q \bar{q}-0}
$$


where, as explained before, $\mathcal{P}_{\sigma}^{\prime}(\sigma=\tau, s)$ denotes the contributions to the action of plaquettes with base on a fixed time slice. In the continuum limit $\hat{\beta}\left(-\mathcal{P}_{\tau}^{\prime}+\mathcal{P}_{s}^{\prime}\right)$ can be identified with the Minkowski field energy measured in lattice units. Thus for $a \approx 0$,

$$
\hat{\beta}<-\mathcal{P}_{\tau}^{\prime}+\mathcal{P}_{s}^{\prime}>_{q \bar{q}-0} \approx a \sum_{\vec{x}} a^{3} \frac{1}{2}<-\vec{E}^{2}(\vec{x})+\vec{B}^{2}(\vec{x})>_{q \bar{q}-0}
$$

where $\left(-\frac{1}{2} \vec{E}^{2}\right)$ and $\frac{1}{2} \vec{B}^{2}$ are the electric and magnetic contributions to the (Minkowski) field energy densities expressed in terms of the euclidean fields. Now the field energy, after subtracting the self energy contributions, is expected to be related to the potential by

$$
V(R)=\frac{1}{2} \int d^{3} x<-\vec{E}^{2}(\vec{x})+\vec{B}^{2}(\vec{x})>_{q \bar{q}-0}
$$

Hence on the lattice $\frac{1}{a} \hat{V}\left(\frac{R}{a}, \hat{\beta}(a)\right)$ should also match the total field energy for $a \rightarrow 0$. Thus if (24) and (25) holds, then we are led to conclude that the second term appearing on the lhs of (24) should vanish in this limit. This is the case if either $\hat{V}+\hat{R} \frac{\partial \hat{V}}{\partial \hat{R}}=0$, $\partial \ln a / \partial \hat{\beta}=0$, or $\eta_{+}=0$. In the first case $\hat{V}(\hat{R}, \hat{\beta})$ has the form $\hat{V} \sim \alpha(\hat{\beta}) / \hat{R}$, and hence does not allow for a confining potential. The second possibility is clearly excluded for $S U(N)$ gauge theories. Hence we conclude that in a confining theory like QCD, $\eta_{+}$would have to vanish for $\hat{\beta} \rightarrow \infty$. But according to (20) and (15),

$$
\eta_{+}=N\left\{\left(\frac{\partial g_{\tau}^{-2}}{\partial \xi}\right)_{\xi=1}+\left(\frac{\partial g_{s}^{-2}}{\partial \xi}\right)_{\xi=1}\right\}
$$

Hence the above line of reasoning would lead to the conclusion that the quantity appearing in curly brackets should vanish in the limit $\hat{\beta} \rightarrow \infty$. This disagrees with the result obtained in ref. [3], where this quantity was shown to be given by $b_{0}$, defined in (10). The author was led to this result by requiring the invariance of the string tensions computed from space-time and space-like Wilson loops under changes in the anisotropy parameter $\xi$. On an isotropic lattice the two string tensions extracted in this way should coincide. By making further use of the action sum rule, Karsch was led to the above conclusion. 
Using the value $\eta_{+}=N b_{0}$ obtained in [3], the approximation for $\eta_{-}$given in (23), and the one-loop approximation $\partial \ln a / \partial \hat{\beta}=-\frac{1}{4 N b_{0}}$, one finds that, for a confining potential, (24) reduces to

$$
\hat{\sigma} \hat{R} \approx 2 \hat{\beta}<-\mathcal{P}_{\tau}^{\prime}+\mathcal{P}_{s}^{\prime}>_{q \bar{q}-0}
$$

which, with the identification (25), would violate the energy sum rule (26) by a factor of two. We emphasize that the origin of the factor two in (27) is the second term appearing on the rhs of (21). With $\eta_{-} \approx \hat{\beta}=2 N / g_{0}^{2}$, eq. (21) takes the form

$$
\hat{V}(\hat{R}, \hat{\beta}) \approx \frac{2 N}{g_{0}^{2}}\left\{<-\mathcal{P}_{\tau}^{\prime}+\mathcal{P}_{s}^{\prime}>_{q \bar{q}-0}-\frac{g_{0}^{2}}{2 N} \eta_{+}<\mathcal{P}_{\tau}^{\prime}+\mathcal{P}_{s}^{\prime}>_{q \bar{q}-0}\right\} .
$$

The contribution proportional to $\left\langle\mathcal{P}_{\tau}^{\prime}+\mathcal{P}_{s}^{\prime}>_{q \bar{q}-0}\right.$ is absent in the expression obtained in [4]. The energy sum rule would have the naively expected form, $\hat{V}=\hat{\beta}<-\mathcal{P}_{\tau}^{\prime}+\mathcal{P}_{s}^{\prime}>_{q \bar{q}-0}$, if one neglects this apparent perturbative contribution. The action sum rule (13) for a confining potential, together with the value $\eta_{+}=N b_{0}$ taken from [3], however tells us that this contribution is about $\frac{1}{2} \hat{V}$ for a confining potential.

Summarizing, our above discussion has shown, that if the weak coupling relations (16) are used to identify the rhs of (22) with the field energy in the continuum limit, then the implementation of the energy sum rule (26) on the lattice demands that $\eta_{+}$vanishes in this limit, which contradicts the coupling constant sum rule obtained in [3]. This latter sum rule has however been derived without invoking perturbation theory. We are therefore rather tempted to conclude that the weak coupling argument leading to (24) with the identification (25) is inadequate, and that the lattice expression for the field energy is that defined by the right hand side of (21). This stands in sharp contrast to the standard belief, that the field energy is related to $\left\langle-\mathcal{P}_{\tau}^{\prime}+\mathcal{P}_{s}^{\prime}\right\rangle_{q \bar{q}-0}$, which as we have seen only holds for a $1 / R$-potential. For a linearly rising potential, on the other hand, the contribution proportional to $\left\langle\mathcal{P}_{\tau}^{\prime}+\mathcal{P}_{s}^{\prime}>_{q \bar{q}-0}\right.$ is essential, and implies that the rhs of (22) approaches in the continuum limit only one half of the field energy stored in the flux tube connecting the quark-antiquark pair, irrespective of the $S U(N)$ gauge group. Clearly the lattice energy 
sum rule deserves further investigations. In particular it would be of interest to determine

the $\hat{\beta}$-dependence of $\eta_{-}$from Monte Carlo calculations of Wilson loops on anisotropic lattices.

\section{Acknowledgments}

We are very grateful to R. Banerjee, H.G. Dosch, O. Nachtmann, and in particular to I.O. Stamatescu for useful discussions and valuable comments.

\section{REFERENCES}

[1] J. Kuti, J. Polonyi and K. Szlanchanyi, Phys. Lett. 98B (1981) 199; J. Engels, F. Karsch, I. Montvay and H. Satz, Phys. Lett. 101B (1981) 89

[2] A. Hasenfratz and P. Hasenfratz, Nucl. Phys. B193 (1981) 210

[3] F. Karsch, Nucl. Phys. B205 [FS 5](1982) 285

[4] C. Michael, Nucl. Phys. B280 [FS 18] (1987) 13

[5] H.G. Dosch, O. Nachtmann and M. Rueter, Heidelberg preprint HD-THEP-95-12

[6] For some early work see: R. Sommer, Nucl. Phys. B291 (1987) 673; ibid B306 (1988) 180. R.W. Haymaker and J. Wosiek, Phys. Rev. Rapid Comm. D36 (1987) 3297; R.W. Haymaker, Y. Peng, V. Singh and J. Wosiek, Nucl. Phys. (Proc. Suppl.) B17 (1990) 558; R.W. Haymaker, V. Singh and J. Wosiek, Nucl. Phys. (Proc. Suppl.) B20 (1991) 207; R. Haymaker and J. Wosiek, Phys. Rev. D 43 (1991) 2676 